\title{
Determinación de las constantes ópticas de películas delgadas de óxido de estaño y de plata para su utilización en recubrimientos multicapa de baja emisividad
}

\author{
R.J. MARTÍN PALMA ,J.M. MARTÍNEZ DUART y A. MALATS I RIERA ${ }^{1}$ \\ Departamento de Física Aplicada C-12, Universidad Autónoma de Madrid. 28049 Cantoblanco. Madrid. España. \\ ${ }^{1}$ Centro de Investigación y Desarrollo (CIDA), Cristalería Española S.A., Avda. de Lugo 110, 33400 Avilés. España.
}

\begin{abstract}
La determinación de los valores de las constantes ópticas ( $n$, índice de refracción y $k$, coeficiente de extinción) en el espectro visible de películas tanto de plata $(\mathrm{Ag})$ como de óxido de estaño $\left(\mathrm{SnO}_{2}\right)$, es una condición necesaria para predecir el comportamiento de un recubrimiento multicapa de baja emisividad con la estructura $\mathrm{SnO}_{2} / \mathrm{Ag} / \mathrm{SnO}_{2}$ en relación al confort visual. Para este propósito, se han caracterizado láminas delgadas de $\mathrm{Ag}$ y de $\mathrm{SnO}_{2}$ con un espesor similar al que tienen en los recubrimientos multicapa (90 Å y $380 \AA$ ̊ respectivamente), evitando así la dependencia con el espesor de las constantes ópticas. Se ha utilizado un programa informático de simulación basado en el método matricial para determinar los valores de las constantes ópticas de dichas películas en el espectro visible. Ha sido determinada asimismo la dependencia del coeficiente de absorción $(\alpha)$ con la longitud de onda, así como el valor del ancho de banda prohibida del óxido de estaño, y la naturaleza de la transición óptica.
\end{abstract}

Palabras clave: Índice de refracción, emisividad, plata, óxido de estaño, recubrimiento multicapa.

Determination of the optical constants of tin oxide and silver thin films for their use in multilayer low-emissivity coatings.

The determination of the values of the optical constants ( $n$, refractive index and $k$, extinction coefficient) in the visible range of both silver $(\mathrm{Ag})$ and tin oxide $\left(\mathrm{SnO}_{2}\right)$ films is a necessary condition to predict the behaviour of a $\mathrm{SnO}_{2} / \mathrm{Ag} / \mathrm{SnO} \mathrm{multilayer}_{2}$ for low emissivity coatings, improving the visual comfort. For this purpose, thin films of $\mathrm{Ag}$ and $\mathrm{SnO}_{2}$ with a thickness similar to that of multilayer coatings ( $90 \AA$ and $380 \AA$ respectively) were used in order to avoid the thickness dependence of the optical constants. A simulation computational program based on the matrix method was employed to estimate the values of the optical constants of these films in the visible range. It has been also determined the dependence on the wavelength of the absorption coefficient $(\alpha)$, as well as the value of the energy gap, and the nature of the optical transitions.

Keywords: Refractive index, emissivity, silver, tin oxide, multilayer coating.

\section{INTRODUCCIÓN.}

En la actualidad, los recubrimientos multicapa de baja emisividad basados en plata depositados sobre vidrio arquitectónico, son ampliamente utilizados con el fin de ahorrar energía en el acondicionamiento térmico de los edificios. Estos recubrimientos combinan una alta transmitancia sobre la mayor parte del espectro solar $(0.35-2.5 \mu \mathrm{m})$ con una alta reflectancia sobre el espectro infrarrojo de un cuerpo negro a temperatura ambiente $(3-50 \mu \mathrm{m})$. En particular, hemos demostrado previamente (1) que el recubrimiento multicapa basado en una película muy fina de plata $(\sim 90 \AA$ A $)$ rodeada por por dos películas de óxido de estaño ( $380 \AA$ ) (2) proporciona un buen aislamiento térmico además de unas buenas propiedades ópticas en el rango visible (400-700 $\mathrm{nm}$ aproximadamente). La determinación precisa de los valores de las constantes ópticas ( $n$, índice de refracción y $k$, coeficiente de extinción) en el rango visible de películas de $\mathrm{SnO}_{2}$ y Ag es, entre otras, una condición necesaria para predecir el comportamiento de todo el recubrimiento de baja emisividad. Es bien sabido que las propiedades ópticas de una lámina delgada dependen en gran medida del proceso de deposición empleado para obtener la película. De esta forma, la estructura granular externa, junto con las distribuciones de defectos internos, cavidades o porosidades, frecuentemente determinan muchas de las propiedades ópticas de las películas, además de las propiedades magnéticas, eléctricas y mecánicas (3). Por otra parte, las constantes ópticas de una lámina delgada frecuentemente también dependen del espesor de la película. Por todas estas razones, para predecir el comportamiento de un recubrimiento óptico multicapa es necesaria una precisa determinación de los valores de las constantes ópticas de las películas que componen el recubrimiento, en lugar de utilizar valores tabulados de las constantes ópticas.

Uno de los métodos más ampliamente utilizados para determinar los valores de las constante ópticas $(n$ y $k$ ) de sustratos cubiertos por láminas delgadas, son las medidas de transmitancia $(T)$ y refletancia $(R)(4)$. En el presente trabajo, se han obtenido los espectros experimentales de películas de $\mathrm{SnO}_{2} \mathrm{y}$ de $\mathrm{Ag}$ depositados mediante pulverización catódica sobre vidrio y, a partir de éstos, se han determinados los valores de las constantes ópticas, $n$ y $k$, mediante un programa informático de simulación. Para este fin, se ha empleado un modelo teórico desarrollado en un trabajo previo (5) basado en el método matricial. 


\section{MODELO TEÓRICO.}

Para obtener las expresiones correspondientes a la transmitancia y a la reflectancia, el formalismo aquí empleado está basado en el método matricial, en el que una película delgada es considerada una lámina plano-paralela de extensión infinita, caracterizada por un índice de refracción $n$ y por un coeficiente de extinción $k$ (5). De acuerdo con este método, cada intercara y lámina homogénea (i) en un recubrimiento multicapa puede ser descrita a través de su matriz apropiada, $M_{i}$, que será función del espesor de la película y del índice de refracción (o de los índices efectivos, p- y s-, para el caso de incidencia no normal) $\left(^{6}\right)$. A partir de éstos, la matriz resultante $M_{R}$ de una serie de láminas paralelas vendrá dada por $M_{R}=M_{1} \times M_{2} \ldots\left({ }^{7}\right)$, por lo que el resultado final vendrá dado en función de un producto de un conjunto de matrices $2 \times 2$. En nuestro formalismo, de acuerdo con las condiciones experimentales del presente trabajo, se realizan las siguientes consideraciones (5):

1. Sustrato finito e incoherente para incluir la reflexión de la superficie trasera y permitir el uso de sustratos débilmente absorbentes.

2. Determinación previa del índice de refracción $n$ y del coeficiente de extinción $k$ del sustrato en función de la longitud de onda y del espesor.

3. Utilización de los ángulos de incidencia experimentales para el cálculo de $R$ y $T$, evitando la habitual asunción de incidencia normal.

4. Implementación de un parámetro, $\Delta \mathrm{d}(\mathrm{x}, \mathrm{y})$, que reproduce la pérdida parcial de fase como consecuencia de inhomogeneidades laterales de la película.

Este modelo evita algunas de las simplificaciones habituales (láminas homogéneas, sustrato semi-infinito, ángulo normal de incidencia para medidas de reflectancia, etc.) así como el uso de criterios de continuidad y de suavidad de las constantes ópticas, lo que aumenta la potencia del método en su aplicación a los casos prácticos habituales.

\section{PROCEDIMIENTO EXPERIMENTAL.}

Se ha utilizado un sistema de pulverización catódica para depositar los recubrimientos de óxido de estaño y de plata sobre vidrio. Las películas de $\mathrm{SnO}_{2}$, de un espesor aproximado de $380 \AA$, fueron depositadas en una atmósfera compuesta por $\mathrm{O}_{2} / \mathrm{N}_{2}-\mathrm{H}_{2} /$ Ar a partir de cátodos de estaño mediante pulverización catódica reactiva. Las películas de Ag fueron depositadas mediante pulverización catódica en una atmósfera de Ar y el espesor de las películas de plata es de, aproximadamente, 90 Å. Estos espesores de las películas de plata y de óxido de estaño se seleccionaron de acuerdo a los valores típicos para su aplicación en recubrimientos multicapa de baja emisividad. Las deposiciones se realizaron a presiones entre $10^{-3}$ y $5 \times 10^{-3}$ Torr. Los valores de reflectancia y de transmitancia en el espectro visible (400-700 $\mathrm{nm}$ aproximadamente) fueron medidos mediante la utilización de un espectrofotómetro Hitachi modelo 150-20 de doble haz, equipado con una esfera integradora.

\section{RESULTADOS Y DISCUSIÓN.}

Los valores de las constantes ópticas ( $n$ y $k$ ) de las películas de óxido de estaño y de plata han sido determinadas a partir de los espectros de transmitancia y de reflectancia, que previamente habían sido suavizados empleando el método de la transformada de Fourier ( 25 puntos). Este paso es necesario para evitar el ruido experimental generado durante la adquisición de datos y para mejorar el funcionamiento del programa de simulación. Es necesario, en primer lugar, determinar los valores espectrales de las constantes ópticas del sustrato de vidrio. Los valores obtenidos en el caso del índice de refracción varían entre 1.55 y 1.52 en el espectro visible, decreciendo al aumentar la longitud de onda. El coeficiente de extinción muestra valores menores de $1.6 \times 10^{-6}$ en el intervalo comprendido entre $400 \mathrm{~nm}$ y $700 \mathrm{~nm}$.

Los espectros de reflectancia y transmitancia experimentales y simulados de la película de $\mathrm{SnO}_{2}$ depositada sobre vidrio se presentan en la Figura 1. Puede observarse que existe una muy buena concordancia entre los datos experimentales y los valores obtenidos mediante el programa de simulación empleado en el presente trabajo, que utiliza un método numérico de inversión para resolver el sistema implícito formado por $R, T$, $R_{\text {exp }}$ y $T_{\text {exp }}$ para la obtención de $n$ y $k$ (5). Los valores de $n$ y $k$ correspondientes al óxido de estaño en el espectro visible se han representado en la Figura 2. Como puede observarse, el $\mathrm{SnO}_{2}$ depositado mediante pulverización catódica es un mate-

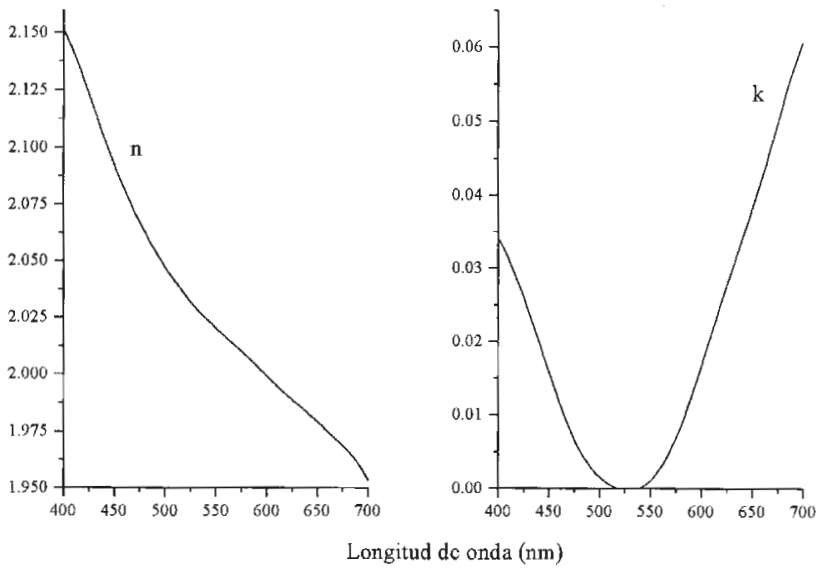

Figura 2. Valores de $n$ y $k$ correspondientes al óxido de estaño en forma de película delgada.
Figura 1. Espectros de reflectancia y de transmitancia experimentales y simulados del óxido de estaño depositado sobre vidrio.

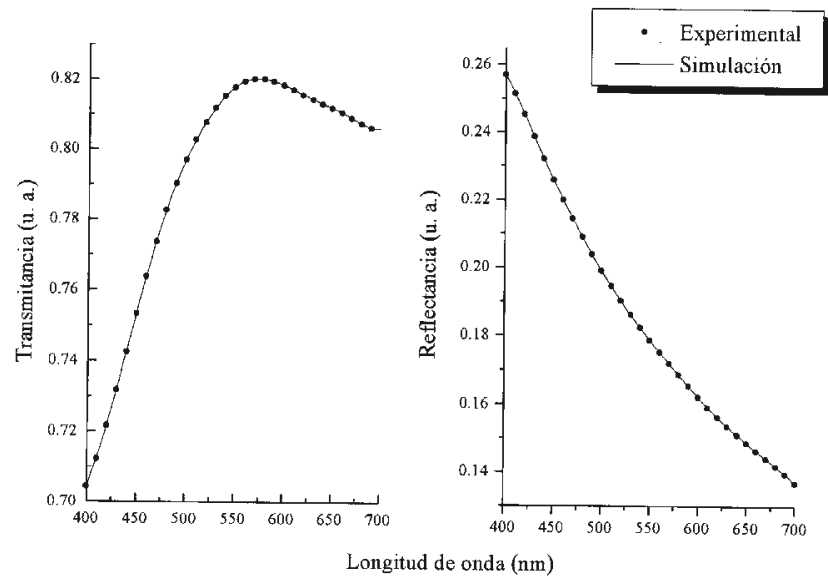

Boletín de la Sociedad Española de Cerámica y Vidrio. Vol. 39 Núm. 4 Julio-Agosto 2000 
rial de alta transparencia en el espectro visible como consecuencia de los bajos valores del coeficiente de extinción. Puede también observarse que la absorción es prácticamente nula en las cercanías de $\lambda=525 \mathrm{~nm}$. El índice de refracción presenta valores crecientes en el espectro visible desde $n \sim 1.95$ a $700 \mathrm{~nm}$ hasta $\sim 2.15$ a $400 \mathrm{~nm}$, lo que coincide con la mayor reflectancia a menores longitudes de onda.

La Figura 3 muestra los valores correspondientes al coeficiente de absorción $(\alpha)$ para el óxido de estaño. Sus altos valores en los extremos del espectro visible (del orden de $10^{4} \mathrm{~cm}^{-1}$ ) son característicos de transiciones de tipo directo (8). Por tanto, para la determinación del valor del ancho de banda prohibido, se ha representado el valor de $a^{2}$ frente a la energía del fotón (Figura 4). A partir de dicha representación se ha determinado que el valor del ancho de banda prohibida es de $2.70 \mathrm{eV}$, mediante la extrapolación del ajuste lineal de los datos experimentales hasta el eje $\alpha^{2}=0$. Los valores del ancho de banda prohibido del $\mathrm{SnO}_{2}$ reportados están entre $3.40 \mathrm{eV}$ y $3.93 \mathrm{eV}$ (9). Sin embargo, el valor del ancho de banda prohibido correspondiente al SnO son cercanos a $1.18 \mathrm{eV}(10)$ y, por tanto, a partir de los datos experimentales del presente trabajo, puede concluirse que se produce óxido de estaño con la composición $\mathrm{SnO}_{x^{\prime}}$ con $1<\mathrm{x}<2$. La formación de dicho compuesto estará relacionada con la técnica de deposición empleada.

La Figura 5 representa los valores espectrales de transmitancia experimentales y simulados de la película de plata depositada sobre vidrio, así como los de la reflectancia. A partir de los datos simulados se han determinado los valores de las constantes ópticas (Figura 6). Es claramente observable que los valores del coeficiente de extinción de la plata son muy altos, lo que indica que la absorción en dicho metal es muy alta. Por tanto, para aplicaciones en el campo de la óptica, es de gran importancia la obtención de una película muy fina de plata para maximizar la transmisión de luz visible, pero debe ser lo suficientemente gruesa como para ser continua y mantener sus buenas propiedades ópticas. Si la película es demasiado delgada, existirá una alta dispersión de la luz incidente como consecuencia de la estructura de islas y, por tanto, el límite inferior para el espesor de dicha película vendrá dado por la formación de una película continua. Por tanto, el límite superior vendrá dado por la transmisión de luz. Sin embargo, el límite

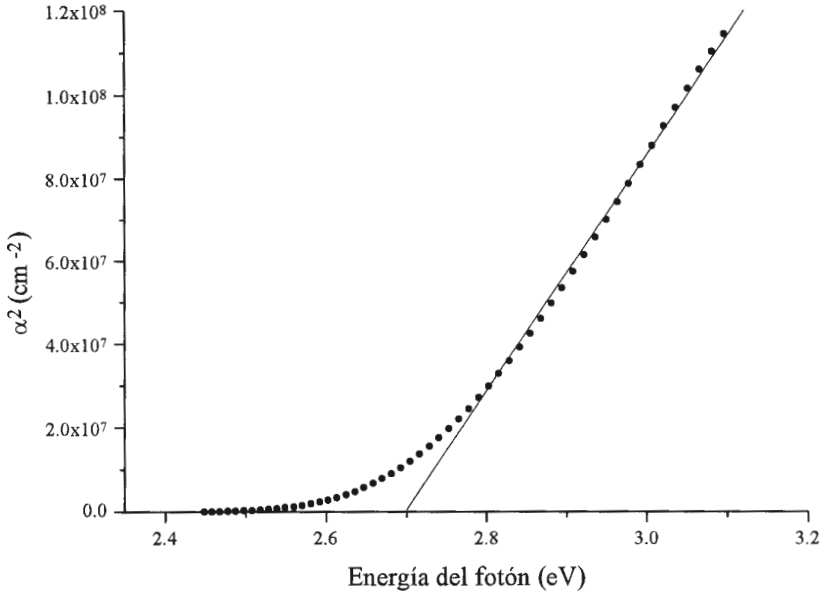

Figura 4. Representación de $\alpha^{2}$ frente a la energía del fotón.

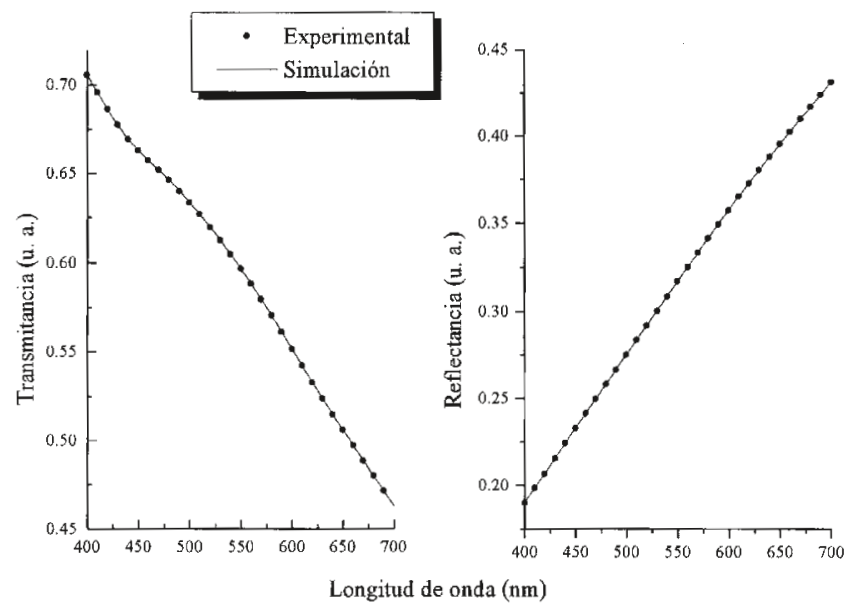

Figura 5. Espectros de reflectancia y de transmitancia experimentales y simulados de la plata depositada sobre vidrio.

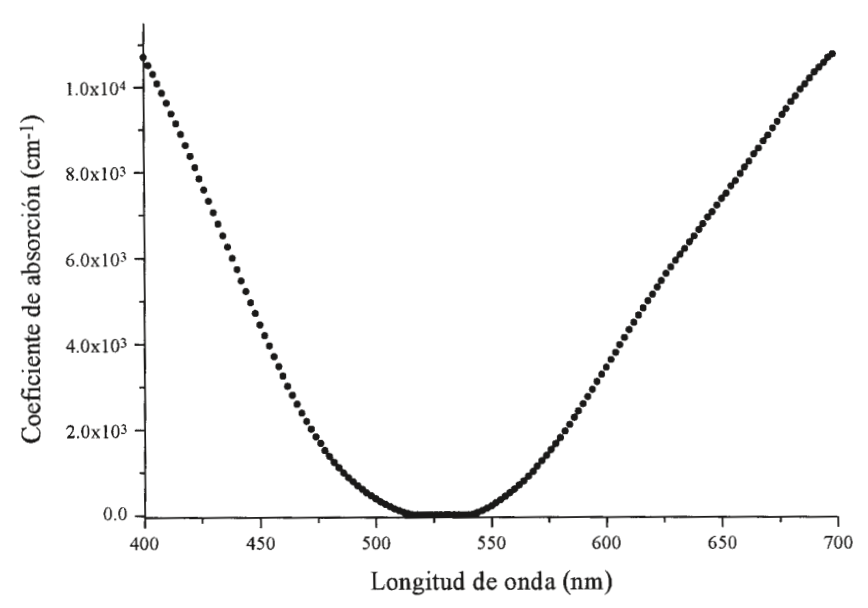

Figura 3. Valores correspondientes al coeficiente de absorción $(\alpha)$ en función de la longitud de onda.

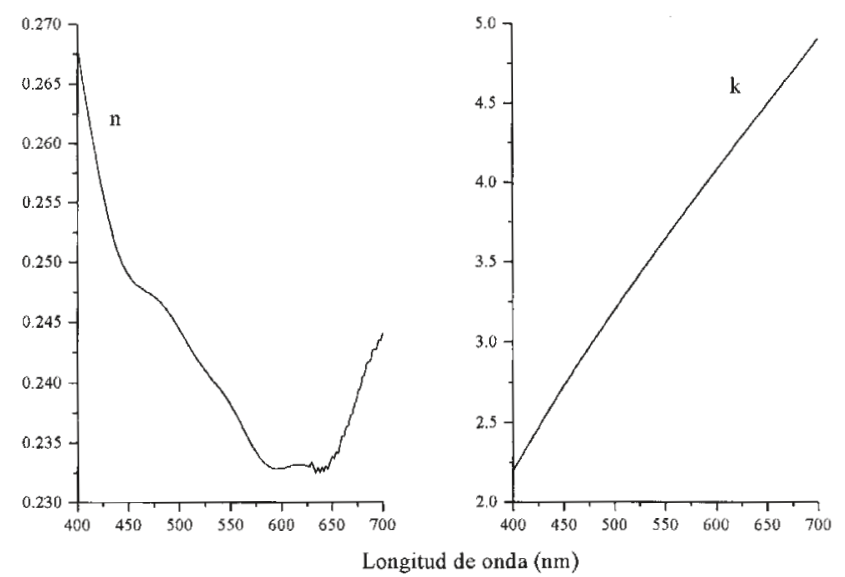

Figura 6. Valores de $n$ y $k$ correspondientes a la plata en forma de película delgada. 
inferior del espesor de la película puede ser reducido depositando otra películas adyacentes a la de plata, lo que facilita un crecimiento bidimensional de la película de plata (por ejemplo $\mathrm{Ni}-\mathrm{Cr}$, etc.). Los valores del índice de refracción de la plata (Figura 6) están situados alrededor de 0.24 y tienen una mínima variación en el espectro visible $(\Delta n \sim 0.03)$. Debe ser notado que los valores de $n$ y $k$ obtenidos para la plata en el presente trabajo son muy cercanos a otros valores publicados (11), como puede observarse en la Figura 7. Las diferencias son, en parte, debidas al bajo espesor de la película de la que se están calculando los valores $n$ y $k$, ya que las propiedades de las películas delgadas son, en general, diferentes a las correspondientes al volumen y dependen ligeramente del espesor, además de la dependencia del método de deposición. Este efecto ha sido previamente estudiado en películas de oro de diferentes espesores depositadas sobre vidrio (12). Los valores experimentales del índice de refracción de la películas de plata aquí obtenidos son mayores que los correspondientes al volumen. Este hecho puede ser asociado a la menor densidad de empaquetamiento de las películas de plata, aumentando la contribución de aire. Los valores del coeficiente de extinción son mayores que los del volumen, que probablemente es debido a la microestructura de la película de plata. En efecto, la microestructura de las películas juega un papel esencial en las propiedades ópticas, teniendo como resultado efectos tales como la dispersión y la absorción anómala que reduce la cantidad de luz transmitida.

\section{CONCLUSIONES.}

Los valores de las constante ópticas ( $n$ y $k$ ) de los componentes más importantes del recubrimiento multicapa de baja emisividad con la estructura $\mathrm{SnO}_{2} / \mathrm{Ni}-\mathrm{Cr} / \mathrm{Ag} / \mathrm{Ni}-\mathrm{Cr} / \mathrm{SnO}_{2}$ han sido determinados empleando un programa informático de simulación basado en el método matricial. Debe ser notado que el modelo óptico empleado en el presente trabajo evita las aproximaciones habituales como lámina homogénea, sustrato semi-infinito, ángulo normal de incidencia para medidas de

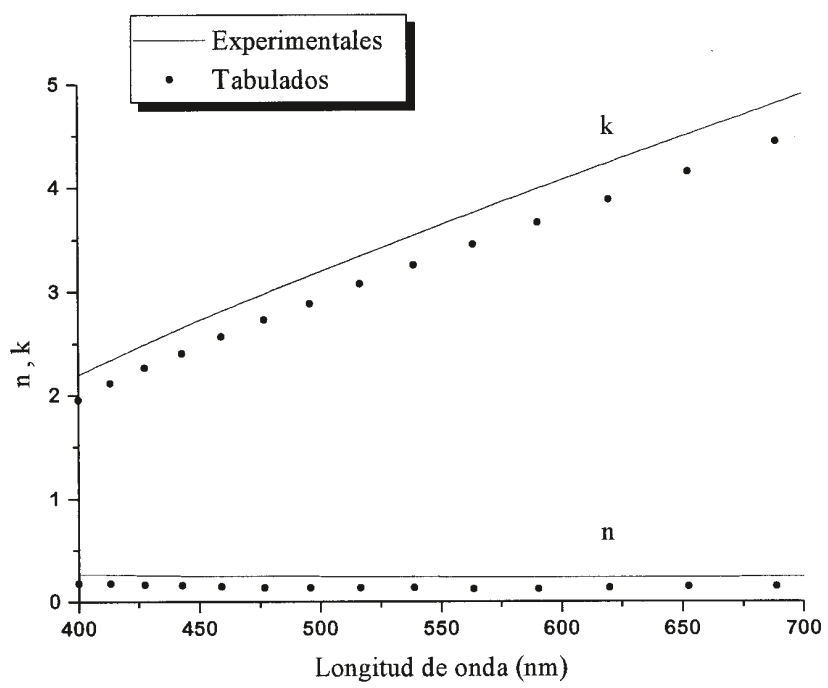

Figura 7. Comparación entre los valores tabulados de $n$ y $k$ de la plata y los valores obtenidos experimentalmente. reflexión, etc., así como el uso de criterios de continuidad y suavidad para las constantes ópticas.

Los valores del coeficiente de extinción determinados en este trabajo para el óxido de estaño depositado mediante pulverización catódica, son los correspondientes a un material de alta transparencia en el espectro visible, siendo la absorción prácticamente nula en las proximidades de una longitud de onda de $525 \mathrm{~nm}$. El índice de refracción crece desde $n \sim 1.95$ a $\lambda=700 \mathrm{~nm}$ hasta $n \sim 2.15$ a $\lambda=400 \mathrm{~nm}$. Por otra parte, los valores correspondientes al coeficiente de extinción de la plata depositada mediante pulverización catódica crece desde aproximadamente 2.2 a $\lambda=400 \mathrm{~nm}$ hasta 4.9 a $\lambda=700 \mathrm{~nm}$, mientras que los valores del índice de refracción están situados alrededor de 0.24 y muestran una mínima variación en este intervalo de longitudes de onda. Las pequeñas diferencias encontradas entre los valores experimentales de la plata y otros valores previamente publicados en películas delgadas y en volumen, son probablemente debidos al crecimiento de la películas de plata como consecuencia del proceso de deposición y el espesor extremadamente bajo de la película de plata. Ha sido también determinado el valor del ancho de banda prohibido del óxido de estaño en forma de lámina delgada, obteniéndose un valor de $2.70 \mathrm{eV}$, y la naturaleza de la transición óptica a partir de la dependencia del coeficiente de absorción con la longitud de onda, resultando ser directa.

\section{BIBLIOGRAFÍA}

1. R.J. Martín-Palma, L. Vázquez, J.M. Martínez-Duart and A. Malats-Riera (Silver-based low-emissivity coatings for architectural windows: optical and structural properies). Solar Energy Matererials \& Solar Cells 53, 55-66 (1998)

2. "Vitrage à couches avec une forte reflexion des rayonnements thermiques". European Patent EP 0506507 B1.

3. M. Ohring. The Materials Science of Thin Films pp.507-545. Academic Press, Inc. San Diego (1992).

4. D.P. Arndt, Applied Optics 23, 3571 (1984)

5. R.M. Bueno, J.F. Trigo, J.M. Martínez-Duart, E. Elizalde and J.M. Sanz. (Study of the optical constants determination of thin films: Dependence on theoretical assumptions). Journal of Vacuum Science \& Technology A13(5), 2378-2383 (1995).

6. O.S. Heavens. Thin Film Physics ( $1^{a}$ ed.) pp. 76-78. Chapman and Hall, London (1970)

7. R.F. Potter. (Basic parameters for measuring optical properties), pp. 14-16 en Handbook of Optical Constants of Solids (1 $1^{\mathrm{a}}$ ed.). Ed. E.D. Palik. Academic Press, Inc. Orlando (1985)

8. I. Mártil and G. González-Díaz. (Undergraduate laboratory experiment: measurement of the complex refractive index and the band gap of a thin film semiconductor). A. J. of Physics 60(1), 83-86 (1992).

9. Z.M. Jarzelski and J.P. Marton. (Physical properties of $\mathrm{SnO}_{2}$ materials. III Optical properties). J. of the Electroc. Soc. 123, 333C-346C (1976).

10. K. Murali Krishna, M. Sharon, M.K. Mishra and V.R. Marathe. (Selection of optimal mixing ratios to obtain suitable photoelectrodes from mixed semiconductors using band gap calculations). Electroch. Acta 41(13), 1999-2004 (1996).

11. D.W. Lynch and W.R. Hunter. (Comments on the optical constants of metals and an introduction to the data for several metals) pp. 353-357 en Handbook of Optical Constants of Solids ( $1^{a}$ ed.). Ed. E.D. Palik. Academic Press, Inc. Orlando (1985).

12. P. Gadenne, Y. Yagil and G. Deutscher. (Transmittance and reflectance in situ measurements of semicontinuous gold films during deposition). J. Appl. Physics 66(7), 3019-3025 (1989). 